\title{
Two solutions for the processing of ambulatory electrocardiogram
}

\author{
Carlos R. Vázquez Seisdedos ${ }^{1}$, João Evangelista Neto ${ }^{2}$, Alexander A. Suárez León ${ }^{3}$, Roberto C. Limão de Oliveira ${ }^{4}$ \\ ${ }^{l}$ Centro de Estudios de Neurociencias, Procesamiento de Imágenes y Señales, Universidad de Oriente, Santiago de Cuba, Cuba. \\ (cvazquez@fie.uo.edu.cu) \\ ${ }^{2}$ Escola Superior de Tecnologia, Universidade do Estado do Amazonas, Manaus, Brasil.(joao_evangelista_neto@yahoo.com) \\ ${ }^{3}$ Departamento de Ingeniería Biomédica, Universidad de Oriente, Santiago de Cuba, Cuba. (aasl@fie.uo.edu.cu) \\ ${ }^{4}$ Faculdade de Engenharia Elétrica, Universidade Federal do Pará, Belém, Brasil. (limao@ufpa.br)
}

\begin{abstract}
The Electrocardiogram (ECG) ambulatory monitoring has three stages: acquisition, processing and analysis, in each one, the information on ECG signal itself or the time series extracted from it are affected by noises, interferences and artifacts. The analysis of the duration and variability of heart intervals establishes rules for the selection of valid beats and to determine with accuracy the fiducially points, especially the $\mathrm{T}$ wave end. This paper describes two problems and their solutions involved in the processing stage. First, a method is described to identify the valid beats for heart rate variability analysis, and then another one is presented for the determination of $\mathrm{T}$ wave end point, based on the calculation of trapezes areas. The validation of both methods exhibits excellent performance indexes in comparison with other approaches.
\end{abstract}

Keywords: ECG, T-wave end, ECG classification, artifacts

\section{Duas soluções no processamento do eletrocardiograma ambulatorial}

\section{RESUMO}

A monitorização do Eletrocardiograma (ECG) ambulatorial tem três etapas: aquisição, processamento e analise, em cada uma, a informação do sinal de ECG o das series temporais extraídas deste está afetada por ruídos, interferências e artefatos. A analise da duração e variabilidade dos intervalos cardíacos estabelece regras para a seleção dos batimentos validos e para determinar com exatidão os pontos característicos, especialmente o final da onda T. Neste artigo é descrito dois problemas e suas soluções envolvidos na etapa de processamento. Primeiro, é descrito um método para identificar os batimentos validos para a analise da variabilidade da freqüência cardíaca, e logo é apresentado outro método para estimar a posição do ponto final da onda $\mathrm{T}$, baseado no calculo de áreas de trapézios. A validação de ambos os métodos exibe excelente métricas de desempenho em comparação com outros métodos.

Palavras Chaves: ECG, final de onda T, classificação de ECG, artefatos.

\section{INTRODUÇÃO}

A monitorização ambulatorial do electrocardiograma (ECG) abrange as seguintes etapas: (1) aquisição, (2) processamento e (3) analise. Cada etapa inclui aspectos de extrema importância, e retos ainda por resolver. A ênfase deste trabalho está na etapa (2) para propiciar as condições visando aumentar a exatidão na etapa (3).

O processamento do ECG ambulatorial inclui os seguintes passos: (a) preprocessamento, (b) detecção de pontos R, (c) segmentação e normalização, (d) extração de caracteristicas, (e) agrupamento, (f) delineação de otras ondas, por exemplo, as ondas $\mathrm{P}, \mathrm{Q}$ e $\mathrm{T},(\mathrm{g})$ cálculo das series temporais asociadas a intervalos cardiacos, por exemplo: as series $\mathrm{RR}$ (entre picos de ondas $\mathrm{R}$ consecutivos) e RTe (do pico da onda $R$ a final da onda $T$ ). O processamento inclui o uso de métodos de inteligência computacional (passos (c) a (e)) para identificar os batimentos validos ou normais. Os batimentos que forem reconhecidos como artefatos (fisiológicos ou técnicos) serão excluídos da analise. A classificação de arritmias atriais ou ventriculares é um tema amplamente abordado [1] mas não a classificação de batimentos que constituírem artefatos a efeitos da analise da variabilidade da frequencia cardiaca (HRV) e da repolarizacão ventricular (QTV). Este tipo de enfoque não é usual na bibliografia. Dentre os métodos de extração de características mais usados estão o PCA linear e a transformada discreta do cosseno (DCT). O método de

ITEGAM - JETIA Vol. 01, N 02. Junho de 2015. Manaus - Amazonas, Brasil. ISSN 2447-0228 (ONLINE). 
agrupamento mais usado é o Perceptron multicamada (MLP). Em menor proporção (em ordem descendente) são empregados os seguintes métodos de agrupamento: Maquina de suporte vetorial (SVM), Quantificação vetorial adaptativa (LVQ) e Funções de base radial (RBF).

Outro aspecto chave na análise da QTV, é que a presença de ruídos no ECG ambulatorial provoca inexatidoes na determinação do final da onda $\mathrm{T}$ (ponto Te). Ao respeito, a revisão do estado da arte mostra as seguintes limitações:

- Os métodos para estimar o ponto Te nao analisam seus desempenhos em presença de ruído. A única exceção [2] só experimenta com sinais sinteticos de forma qualitativa,

- Em outros casos, a influência do ruído nao é analisada no ponto Te senão em intervalos diferenciais que incluiem ele, por exemplo, RTe, e o numero de morfologias é muito pequeno [3][4].

- Muitos métodos de estimação empregan regras de decisão baseadas em limiar [5][6] que são muito dependentes de ruido.

O objetivo deste artigo é apresentar duas soluções aos seguintes aspectos de processamento do ECG ambulatorial:

1. Reconhecimento de batimentos validos para à analise da HRV e QTV.

2. Detecção do final da onda $\mathrm{T}$ (robusta a ruido).

\section{REVISÃO BIBLIOGRÁFICA}

A monitorização ambulatorial do ECG permite seguir as atividades cotidianas do paciente possibilitando o estudo de casos que pudessem ter episódios arrítmicos fatais, incluindo morte cardíaca súbita (SCD, Sudden Cardiac Death). A causa principal da SCD é o infarto agudo de miocárdio (IAM). Estima-se que aproximadamente $75 \%$ dos pacientes que faleceram subitamente tiveram um infarto de miocárdio assintomático [7].

Nos últimos anos foram desenvolvidos diversos métodos e algoritmos para estimar o pico da onda $\mathrm{R}$ ou ponto $\mathrm{R}$ [8] o qual facilitou um amplo estudo das variações fisiológicas e patológicas do intervalo RR (entre picos de ondas $\mathrm{R}$ consecutivas). O mesmo não se pode afirmar para a estimação do ponto final da onda $T$ (Te), e dos intervalos associados, QT (do início da onda Q ao final da onda $\mathrm{T}$ ) ou RTe (desde o ponto R ao ponto Te). O Te é um dos pontos mais difícil de determinar devido à suave transição do sinal ao redor dele, eventualmente contaminado por ruído, como é observad na Figura 1.

A diminuição da variabilidade (desvio padrão) do intervalo RR entre batimentos consecutivos (HRV: Heart Rate Variability) foi sugerido como um preditor independente de SCD em pacientes pós-infarto [9]. O alongamento do intervalo QT foi descrito como um preditor de morte súbita em pacientes pós-infarto e arritmias malignas [10]. A variabilidade temporal (batimento a batimento) do intervalo QT (QTV: QT variability) ou espacial (QTD: QT
Dispersion) são medidas da heterogeneidade da repolarização ventricular e tem sido de grande relevância na predição de taquicardia e fibrilação ventricular, eventos previos à SCD [11].

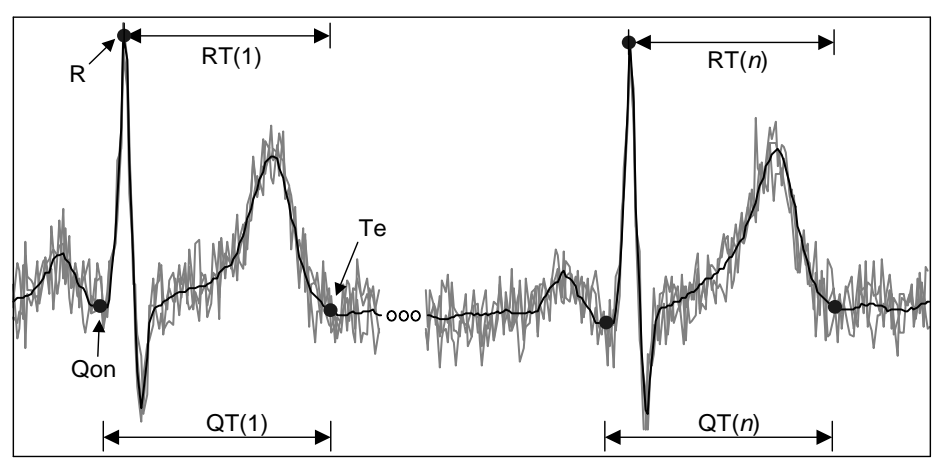

Figura 1. Pontos caracteristicos R e Te e intervalos QT e RT associados à repolarizaçao ventricular em um eletrocardiograma contaminado com ruído de banda larga de origem muscular.

Os valores do desvio padrão das séries QT são pequenos (menores de 20ms) tanto para sujeitos sãos quanto para sujeitos patológicos. Isso cria um desafio para determinar o ponto Te com uma exatidão e precisão tal que seja possível diferenciar pequenas margens de variabilidade. Por exemplo, se o período de amostragem do sinal de ECG for igual a $4 \mathrm{~ms}$, um erro de detecção de 4 amostras (16ms) introduziria um erro significativo no diagnostico usando a variabilidade do QT.

Para melhorar a exatidão e precisão da detecção do ponto Te, nos últimos vinte anos vários métodos e algoritmos têm sido propostos baseados em intersecção de linhas [12], limiar na primeira derivada da onda $\mathrm{T}$ [5], cálculo de: distancias [13], ângulos [14] e áreas [15]; correlação com um padrão [16], uso de: modelos matemáticos [17] e a transformada wavelet [18], dentre outros. De todos eles, os mais reportados nos últimos cinco anos são os algoritmos baseados na transformada wavelet, os quais continuam usando os critérios de limiar nas diferentes escalas da wavelet, o qual aumenta sua sensibilidade ao ruído.

Em situações de alta atividade física, o ruído devido à contrações musculares constitui a principal fonte de erro na estimação do ponto Te (Figura 1) e portanto, da análise da QTV e QTD. Este tipo de ruído possui características espectrais de banda larga e pode ser modelado como ruído branco gaussiano de media zero. A análise da QTV em presença de ruído de banda larga requer uma detecção exata do final da onda $\mathrm{T}$ devido à pequena variabilidade do intervalo QT (ou RT).

A melhora da exatidão e a precisão na estimação do ponto Te em presença deste tipo de ruído e de outros que afetam ao sinal de ECG ambulatorial é um dos tópicos de pesquisa ainda aberto.

$\mathrm{O}$ grande desafio da monitorização ambulatorial é a presença de artefatos devido a causas de origem técnica e fisiológica [19]. Os artefatos no ECG são perturbações que afetam (parcial ou totalmente) à morfologia do sinal eletrocardiográfico. Os algoritmos de detecção de pontos no ECG mais sofisticados falham em presença de artefatos, provocando falsos positivos (artefatos detectados como batimentos) e falsos negativos (batimentos não detectados) nas séries temporais RR, QT e outras. 
Para que a análise da HRV ou QTV seja valida, segundo a comissão denominada como Task Force [20], deve-se verificar que cada onda $\mathrm{R}$ detectada corresponda a um batimento completo que tenha sido originado no nodo sinoatrial e onde não exista nenhum tipo de bloqueio da atividade elétrica quando o impulso elétrico transite pelos ramos direito e esquerdo do feixe de Hiz e suas correspondentes ramificações finais ou fibras de Purkinje (Figura 2). Caso contrário e para fins de estudos da HRV e a QTV, o batimento será considerado como um artefato localizado nas posições correspondentes das séries temporais RR ou QT, respectivamente, e deveria ser excluído da análise.

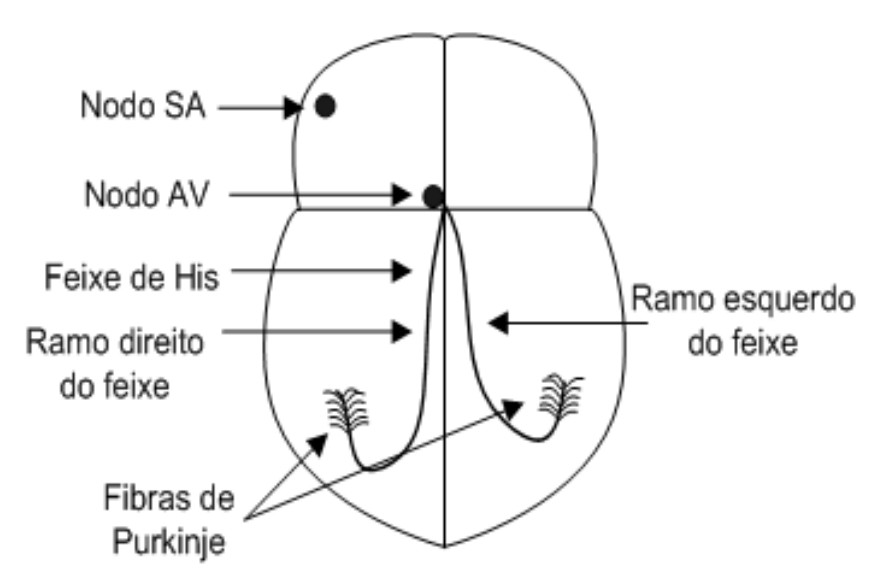

Figura 2. Sistema de condução da atividade elétrica do coração

Os artefatos no ECG podem ser de origem fisiológica e técnica (por exemplo, os artefatos devido ao movimento do paciente). Para atingir o critério anterior é necessário realizar um reconhecimento morfológico automatizado, batimento a batimento no sinal de ECG, que valide os batimentos validos (normais) e não validos (a partir daqui denominados de artefatos). Por exemplo, um extra-sístoles supraventricular possui um padrão muito parecido a um batimento normal, mas não possui onda P. Portanto, se nesse batimento fossem obtidos os pontos $\mathrm{R}$ e Te, e posteriormente o intervalo RTe, estar-se-ia cometendo um erro. O mesmo ocorreria com um artefato de origem técnica que tenha uma morfologia parecida a uma onda $\mathrm{R}$, e seja detectado como tal.

A monitorização ambulatorial expôs um problema que ainda não está completamente resolvido: a obtenção de um diagnóstico que seja robusto à presença de artefatos no ECG, e de falsos positivos e negativos nas séries temporais RR e QT.

Até o presente, muitos trabalhos foram desenvolvidos para o reconhecimento morfológico de batimentos especialmente para diagnosticar diversos tipos de arritmias atriais ou ventriculares [1][21]. A metodologia de validação emprega sinais reais, mas não são usados sinais sintéticos. Visto que o uso destes sinais permite a inclusão de artefatos (fisiológicos e técnicos) de múltiplas morfologias em um batimento, que não são observados nas bases de dados reais disponíveis. Apesar de que os sinais de ECG de bases de dados constituem situações reais, a validação robusta e sensível a rápidas mudanças morfológicas entre batimentos requer uma ampla variedade de morfologias, que também não estão disponíveis nestas bases de dados de sinais. Não foi encontrada nenhuma publicação para classificar batimentos que constituírem artefatos para efeitos da análise da HRV e a QTV. Este tipo de enfoque não aparece reportado na bibliografia.

Os aspectos mencionados nos parágrafos anteriores inspiraram a ideia de desenvolver diversos métodos de processamento de sinal, usando métodos de inteligência computacional, que sejam mais exatos e robustos em presença de elementos que contaminem a informação diagnóstica presente no sinal de ECG nos diversos cenários da monitorização ambulatorial. Especificamente este trabalho centra seu interesse em resolver dois problemas relacionados com a análise do intervalo de repolarização ventricular durante monitorização ambulatorial do ECG. O primeiro é a pesquisa de um método para o reconhecimento de batimentos que constituírem artefatos para efeito do estudo de sua variabilidade. Para essa finalidade são usadas técnicas de inteligência computacional. $O$ segundo é a pesquisa de um algoritmo para a detecção do final da onda $\mathrm{T}$, que seja pouco sensível à presença de ruído branco gaussiano.

\section{MATERIAIS E MÉTODOS}

Nos dois métodos explicados nesta secção pressupõem que os picos das ondas $\mathrm{R}$ foram previamente identificados, o que equivale a empregar a um algoritmo infalível para estimar esse pontos. Neste trabalho serão usadas as marcações dos pontos $R$ realizados pelos especialistas nas bases de dados correspondentes, mas na pratica, pode ser usado qualquer detector $\mathrm{R}$ com suficiente robustez. Em [8] existe uma extensa revisao de metodos para esta finalidade.

\section{A. Reconhecimento de batimentos}

O alvo é pesquisar uma combinação otima de métodos de extração de características e de agrupamento dentre os citados anteriormente. A busca da combinação otima foi realizada seguindo os seguintes passos:

1. Caracterização do desempenho conjunto dos três métodos de extração (DCT, PCA e KPCA) e os quatro classificadores citados (MLP, SVM, LVQ e RBF), realizando uma avaliação parcial com 4000 batimentos reais. O objetivo é investigar o método de agrupamento de melhor desempenho,

2. Avaliação global dos três métodos de EC para o método de agrupamento selecionado no passo 1, usando todos os batimentos da MIT-BIH Arrhythmia Data base (110192 batimentos)

Preprocessamento - foi usado um filtro passafaixa, consistente em um filtro passa-altas (Butterworth, fase zero, 6ta. Ordem, frequencia de corte igual a $0,6 \mathrm{~Hz}$ ) em cascata com um filtro passabaixas (Butterworth, fase zero, 12ma ordem, frequencia de corte igual a $45 \mathrm{~Hz}$ ). Posteriormente, o sinal foi convertido num sinal de variancia unitaria, para garantir invariancia com respeito à amplitude, para qualquer batimento.

Segmentação - Foi empregada uma janela assimetrica de um tamanho fixo aoredor do pico R. O comprimento da janela foi 
igual a 235 amostras (valor máximo) incluindo o ponto R. Para a selecção do numero de amostras à direita e à esquerda do ponto $\mathrm{R}$, foi usado a media da relaçao PR/QT para os valores minimos e maximos. A $360 \mathrm{~Hz}$ os valores resultantes sao 92 amostras à esquerda (39\% do total das amostras) e 142 amostras à direita (61\% do total das amostras).

O desempenho de cada classificador foi avaliado mediante o cálculo de: Especificidade (E), Sensibilidade (S) e Valor Preditivo Positivo (VPP), a partir das tabelas de confusão de cada classificador para as 2 classes definidas: Normal (NRMB) e Artefato (ARTF) como se observa na figura 3.
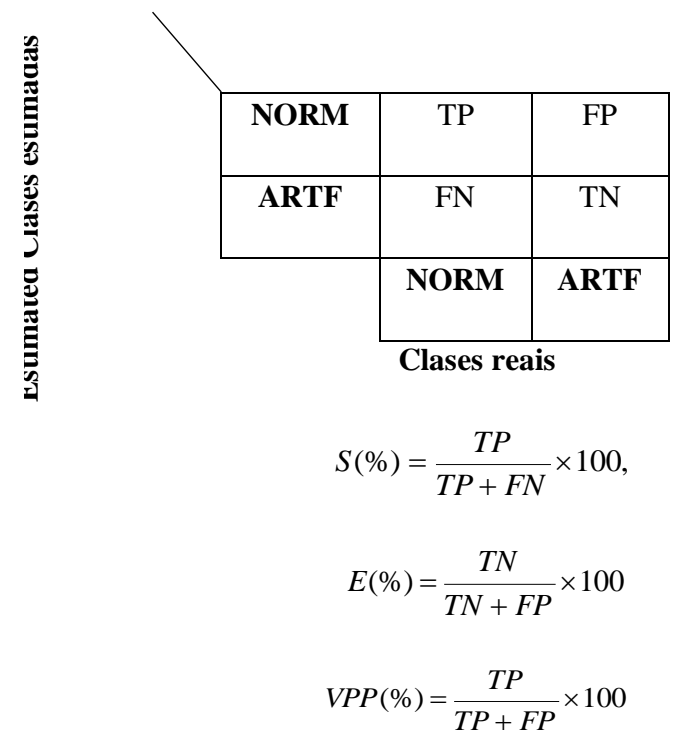

Figura 3. Matriz de confusão para cada classificador. VP: verdadeiros positivos, VN: verdadeiros negativos, FP: Falsos positivos, FN: Falsos negativos, S: Sensibilidade, E: Especificidade, VPP: Valor preditivo positivo.

Para verificar se as diferencias entre os diferentes classificadores são estatisticamente significativas, foi usado o Test de McNemar, que é baseado no calculo do estadígrafo de McNemar, definido como:

$$
z=\frac{\left|n_{01}-n_{10}\right|-1}{\sqrt{n_{01}+n_{10}}}
$$

Sendo:

$n_{01}$ : número de amostras mal classificadas por $\mathrm{A}$ mas não por $\mathrm{B}$. $n_{10}$ : número de amostras mal classificadas por B mas não por A.

\section{B. Detecçao do final da onda $T$}

Para detectar o ponto Te, de forma robusta ao ruído de banda larga foi proposta o método de áreas de trapézios (TRA). O método TRA está baseado no cálculo de áreas sucessivas de trapézios retangulares com dois vértices fixos e dois moveis: $\left(\mathrm{x}_{\mathrm{i}}\right.$, $\left.\mathrm{y}_{\mathrm{i}}\right)$ e $\left(\mathrm{x}_{\mathrm{r}}, \mathrm{y}_{\mathrm{r}}\right)$. O ponto móvel $\left(\mathrm{x}_{\mathrm{i}}, \mathrm{y}_{\mathrm{i}}\right)$ é deslocado pelo sinal de $\left(\mathrm{x}_{\mathrm{m}}\right.$, $\left.\mathrm{y}_{\mathrm{m}}\right) \mathrm{a}\left(\mathrm{x}_{\mathrm{r}}, \mathrm{y}_{\mathrm{r}}\right)$, enquanto a área total é calculada (figura 4).

O final da onda $\mathrm{T}$ será definido como o ponto onde a área A do trapézio é máxima. A fórmula da área do trapézio é:

$$
A=0,5\left(y_{m}-y_{i}\right)\left(2 x_{r}-x_{i}-x_{m}\right)
$$

Sendo:

- $\left(\mathrm{x}_{\mathrm{m}}, \mathrm{y}_{\mathrm{m}}\right)$ a abscissa e a ordenada, respectivamente, de um ponto com a máxima derivada absoluta após o pico da onda $\mathrm{T}$.

- $\left(\mathrm{x}_{\mathrm{r}}, \mathrm{y}_{\mathrm{r}}\right)$ a abscissa e a ordenada, respectivamente, de um ponto de referência localizado no segmento T-P isoelétrico. O local exato não é muito importante contanto que o ponto esteja após o final da onda $\mathrm{T}$.

- $\left(\mathrm{x}_{\mathrm{i}}, \mathrm{y}_{\mathrm{i}}\right)$ é a abscissa e ordenada, respectivamente, de um ponto móvel entre os dois pontos anteriores.

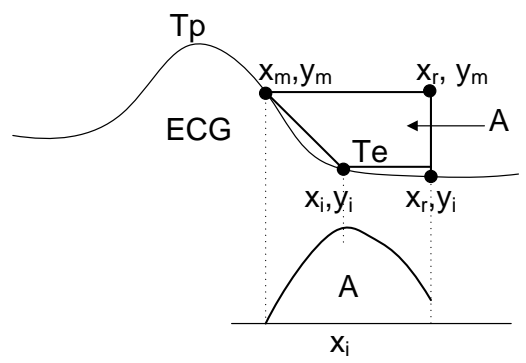

Figura 4. Determinação do final da onda $\mathrm{T}$ (ponto $\mathrm{Te}$ ) para uma onda monofásica mediante o calculo das áreas dos trapézios.

\section{O preprocesamento previo consiste em:}

1. Filtragem passa-altas do sinal do ECG (Butterworth, bidirecional, $4^{\mathrm{a}}$ ordem, à frequência de $0,5 \mathrm{~Hz}$ ) para reduzir as flutuações da linha base.

2. Filtragem passa-baixas do ECG obtido em (1) (Butterworth, bidirecional, $4^{\mathrm{a}}$ ordem, à frequência de $30 \mathrm{~Hz}$ ) para reduzir ruídos e interferências.

A quantidade $z^{2}$ se distribui aproximadamente como $\chi^{2}$ con um grado de libertade. A hipótese nula $H_{0}$ (os dois classificadores têm o mesmo erro) pode ser rejeitada com uma probabilidade de erro de 0,05 se $|z|>1,96$. A hipótese alternativa $H_{1}$ é que os classificadores têm erros distintos. Se o resultado $|z|$ é significativo, então há suficiente evidencia para rejeitar a hipótese nula em favor da hipótese alternativa, o que significa que os classificadores não têm o mesmo erro e, por 
conseguinte as diferencias nos índices de desempenho são estatisticamente significativas. O estadígrafo $|z|$ foi obtido para cada par de classificadores dentre todas as combinações possíveis, para o casso da avaliação global que constitui a população experimental maior e, portanto, o casso mais desfavorável. Para o caso de ondas bifásicas ou somente ascendente ou descendente, o ponto $\left(\mathrm{x}_{\mathrm{m}}, \mathrm{y}_{\mathrm{m}}\right)$ é escolhido de tal modo que a seção da onda entre este ponto e o ponto $\left(\mathrm{x}_{\mathrm{r}}, \mathrm{y}_{\mathrm{r}}\right)$ tenha um tendência monótona crescente ou decrescente.

Desempenho em condições ruidosas - Para caracterizar o desempenho em presença de ruído para cada sinal de ECG da QTDB, foi realizado o seguinte procedimento:

1. Filtragem passa-altas, em seguida passa-baixas, similar ao pré-processamento descrito para o algoritmo TRA.

2. Obtenção do final de onda $\mathrm{T}$ de referência usando $\mathrm{O}$ método X $\left(T_{\mathrm{RX}}\right)$. O sub índice de "X" será " $\mathrm{D}$ " para o método do limiar na primeira derivada [5] de amplo uso; e T para o método de áreas de trapézios.

3. Calculo da amplitude pico da onda $\mathrm{T}$ de referência $\left(\mathrm{A}_{\mathrm{TWP}}\right)$ usando os valores anotados pelos especialistas.

4. Para cada batimento do sinal ECG filtrado, foi adicionado ruido branco (WN) com amplitude igual a um $N \%$ de ATWP, $N=\{3 \%, 5 \%, 10 \%, 20 \%\}$. Para cada nível de ruído, foram geradas 200 realizações de WN e adicionadas ao sinal de ECG filtrado; então foi filtrado passa-baixa conforme a etapa 2 do preprocessamento, e finalmente foi calculada a média dos 200 respectivos finais de onda T. Este valor se torna o final de onda $\mathrm{T}$ para o nível de ruído $\mathrm{N}$ e método $\mathrm{X}\left(T_{\mathrm{NX}}\right)$.

5. Obtenção das estimativas sucessivas do final de onda $\mathrm{T}$ para cada batimento $i$, nível de ruído $N$ e método $X\left(T_{\mathrm{NXi}}\right)$.

6. Calculo do erro relativo percentual modular $\left(E_{\mathrm{NX}}\right)$ para o algoritmo " $X$ " e nível de ruído $N$ segundo a expressão seguinte:

$$
E_{N X}=\overline{\sum_{i=1}^{k}\left|\left(\frac{T_{N X i}-T_{R X i}}{T_{R X i}}\right)\right|} \times 100
$$

Onde:

- $k$ é o número total de batimentos anotados por ambos os cardiologistas (3112).

- $T_{\mathrm{NXi}}$ é o $i$-esimo final de onda T obtido pelo algoritmo $X$ quando o nível de ruído é $N \%$ de $A_{\text {Twp. }} \quad N=\{3 \%, 5 \%, 10 \%, 20 \%\}$ referendado ao valor máximo da onda $\mathrm{T}$ (ponto $\mathrm{Tm}$ ) .

- $T_{\mathrm{RXi}}$ é o i-esimo final de referência de onda $\mathrm{T}$ para o algoritmo $X$, referendado ao ponto $\mathrm{Tm}$. Para o algoritmo de limiar na primeira derivada (THD), são usados os fatores de limiar seguintes: $K=2(50 \%)$ e $K=5(20 \%)$.

$\mathrm{O} E_{\mathrm{NX}}$ é a média global dos erros de detecção relativo modulares devidos ao ruído somado. Este parâmetro dá uma idéia do deslocamento para diante ou para trás (ou seja, absoluto) da posição do final da onda T devido ao efeito do ruído. Assim, $E_{\mathrm{NX}}$ é uma medida do desempenho de método em condições ruidosas.

O processamento dos sinais foi realizado com Matlab 7.7 (The Math-Works, Inc, Natick, MA, 2008).

\section{RESULTADOS E DISCUSSÕES}

\section{A. Reconhecimento de batimentos}

Avaliação parcial - Das 36 combinaçoes possiveis, o classificador MLP obteve os melhores resultados, para todas as variantes de extraçao de caracteristicas, tanto em treinamento quanto a validaçao. A figura 5 apresenta os valores da Especificidade obtidos. O desempenho foi similar para os outros indicadores [21].

Avaliação global - Na tabela 1 apresenta os resultados da avaliação para 110192 batimentos da base de dados. Só foram excluídos 96 batimentos, o primeiro e o último de cada registro. Para 10 componentes, o método PCA + MLP supera em especificidade e valor preditivo positivo aos outros dos métodos, embora é menos sensível que DCT + MLP e KPCA + MLP, nessa ordem. Para 15 e 20 componentes, o método KPCA + MLP é superior a DCT + MLP e PCA + MLP, apresentando desempenhos maiores que (o iguais a) os melhores obtidos pelos outros dois métodos.

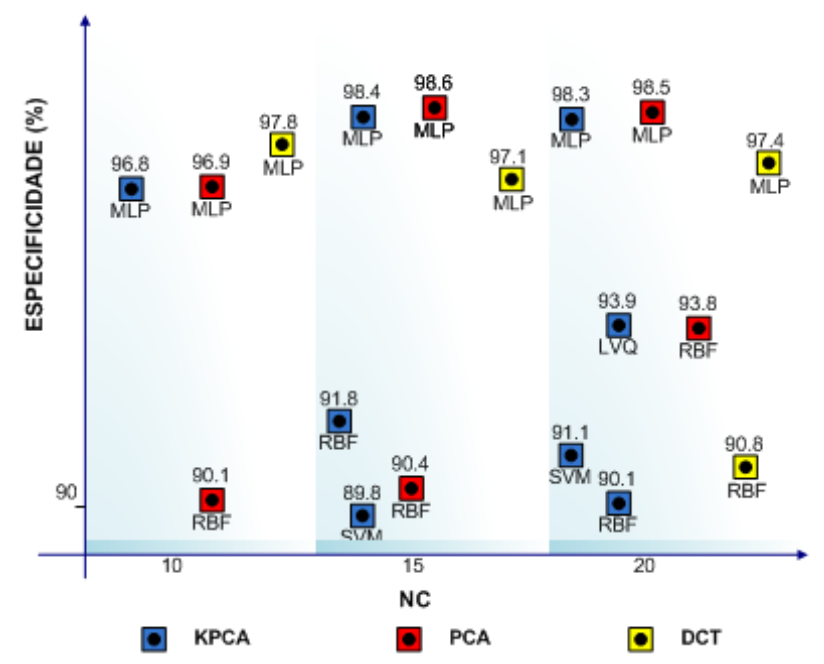

Figura 5. Índice de especificidade para cada classificador.

Tabela 1. Todos os classificadores usando MLP.

\begin{tabular}{|c|c|c|c|c|c|c|c|c|c|}
\hline \multicolumn{10}{|c|}{ Validação } \\
\hline \multirow{2}{*}{ Classif. } & \multicolumn{3}{|c|}{ DCT } & \multicolumn{3}{|c|}{ PCA } & \multicolumn{3}{|c|}{ KPCA } \\
\cline { 2 - 11 } & $\mathbf{1 0}$ & $\mathbf{1 5}$ & $\mathbf{2 0}$ & $\mathbf{1 0}$ & $\mathbf{1 5}$ & $\mathbf{2 0}$ & $\mathbf{1 0}$ & $\mathbf{1 5}$ & $\mathbf{2 0}$ \\
\hline $\boldsymbol{S p}(\boldsymbol{\%})$ & 94 & 95 & 96 & 95 & 96 & 97 & 95 & 96 & 97 \\
\hline $\boldsymbol{S e}(\boldsymbol{\%})$ & 95 & 95 & 95 & 94 & 95 & 94 & 94 & 95 & 95 \\
\hline $\boldsymbol{P P V}(\boldsymbol{\%})$ & 97 & 98 & 98 & 98 & 98 & 98 & 97 & 98 & 98 \\
\hline
\end{tabular}


Custo computacional - A custo computacional médio de KPCA + MLP é 1094 vezes maior que PCA+MLP, e 370 maior que DCT + MLP.

Os valores do estadigrafo $|z|$ estao entre 5,6 e 222,3. Assim, para todos os classificadores, pode ser rejeitado a hipótese nula e por tanto é possível estabelecer que as diferencias encontradas nos índices de rendimento para cada um dos classificadores propostos são estatisticamente significativas. Não foi possível comparar os resultados com outros estudos sobre reconhecimento de artefatos, devido a que não existem (ate nosso conhecimento).

\section{B. Detecção do final da onda $T$}

Desempenho em condições ruidosas - A avaliação do metodo de areas de trapezios usando a base de dados QTDB mostrou excelentes resultados de exatidão e precisão [22].

A figura 6 apresenta os valores do erro relativo percentual modular $\left(E_{\mathrm{NX}}\right)$ para cada algoritmo e nível de ruído. Em todos os casos, o erro do algoritmo TRA é menor que o obtido com o algoritmo THD com uma alta significação $\left(p \leq 8,9 \times 10^{-7}\right.$ ou até menor). Este resultado mostra o melhor desempenho do algoritmo TRA em presença de níveis baixos, meio e altos de ruído.[

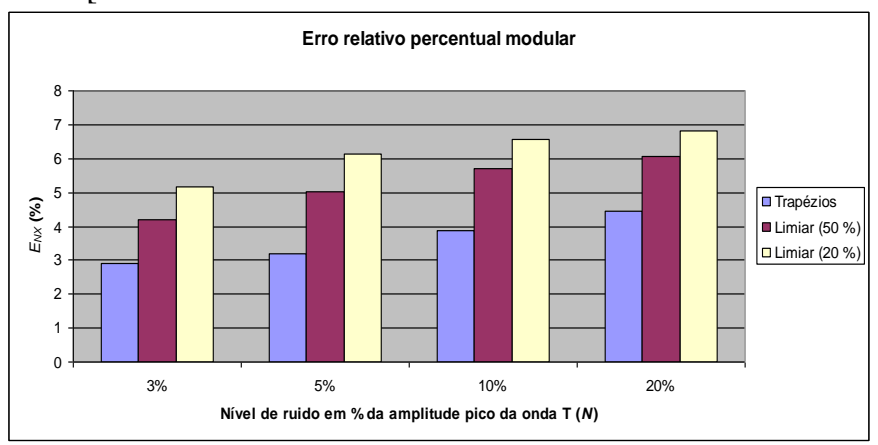

Figura 6. Erro relativo percentual modular versus nível de ruído para os algoritmos: TRA e THD. O valor dos diversos testes de hipótese (tstudent) para cada algoritmo com respeito ao algoritmo dos trapézios está demarcado por uma probabilidade $\mathrm{p} \leq 8,9 \times 10 \square 7$.

Para o limiar de 50\%, o erro médio do algoritmo THD é o menor, corroborando com os resultados apresentados em [5] e é mais alto para o limiar $K=5(20 \%)$. Nos experimentos de Laguna [5], era só considerado o caso com $K=2$ (50\%) porque experimentalmente mostrou o melhor desempenho. Não obstante para $K=2$, o ponto Te fica mais longe do verdadeiro final.

\section{CONCLUSÃO}

$\mathrm{O}$ compromiso entre os indicadores de desempenho e o custo computacional sugere usar a combinaçao KPCA (extração de caracteristicas) e MLP (agrupamento). A pessar de seu maior custo computacional, o método KPCA pode ser implementado con recursos relativamente modestos levando em conta as tecnologias computacionais actuais. A futura melhora e otimização do algoritmo KPCA poderia asegurar maior eficiencia e velocidade, por exemplo, usando dispositivos programaveis.

Para detectar o final da onda $\mathrm{T}$ em sinais ambulatoriais com alto nivel de ruido branco, se sugere usar o algoritmo dos trapecios pela robustez apresentada, alem da vantagem de nao empregarem nenhum fator empírico para seu funcionamento.

\section{REFERÊNCIAS BIBLIOGRÁFICAS}

[1] Ceylan, R., Özbay, Y. Comparison of FCM, PCA and WT techniques for classification ECG arrhythmias using artificial neural network. Expert Systems with Applications, 33, pp. 286-295, 2007.

[2] Martinez A, Alcaraz R, Rieta JJ: Application of the phasor transform for automatic delineation of single-lead ECG fiducial points. Physiol. Meas. 2010, 31: 1467-1485

[3] Porta A, Baselli G, Lambardi F, Cerutti S, Antolini R, Del Greco M, Ravelli F, Nollo G. Performance assessment of standard algorithms for dynamic R-T interval measurement: Comparison between R-Tapex and R-Tend approach, Med. Biol. Eng. Comput. 1998, 36: 35-42.

[4] Tikkanen PE, Sellin LC, Kinnunen HO, Huikuri H V. Using simulated noise to define optimal QT intervals for computer analysis of ambulatory ECG. Medical Engineering \& Physics 1999, 21: 15-25.

[5] Laguna P, Thakor NV, Caminal P, Jane R, Yoon HR, Bayes de Luna A. New algorithm for QT interval analysis in 24-hour Holter ECG: performance and applications. Med Biol Eng Comput. 1990, 28: 67-73.

[6] Martınez JP, Almeida R, Olmos S, Rocha A P, Laguna P. A WaveletBased ECG Delineator: Evaluation on Standard Databases. IEEE Trans. Biomed. Eng. 2004, 51: 570-581.

[7] Sociedade Brasileira de Cardiologia. III Diretriz sobre tratamento do infarto agudo do miocárdio, 2004. Disponível em http://www.scielo.br/scielo.php?script=sci_arttext\&pid=S0066782X2004002200001. Acesso em: 25 mar. 2012.

[8] Köhler, B. U., Hennig, C., Orglmeister, R. The Principles of Software QRS Detection. Reviewing and Comparing Algorithms for Detecting this Important ECG Waveform. IEEE Engineering in Medicine and Biology, pp. 42-57, 2002.

[9] Kleiger, R.E.; Miller, J.P. ; Bigger, J.T.; Moss, A.J. Decreased heart rate variability and its association with increased mortality after acute myocardial infarction. Am. J. Cardiol, 59, 1987, pp. 256-262.

[10]. Schwartz P.J., Wolf S. QT interval prolongation as predictor of sudden death in patients with myocardial infarction. Circulation, 57, 1978, p. 1074-1079.

[11] Haigney M.C., Zareba W., Gentlesk P.J., Goldstein R.E., Illovsky M., Mcnitt S., Andrews M.L., Moss A.J., The madit II investigators. QT Interval Variability and Spontaneous Ventricular Tachycardia or Fibrillation in the Multicenter Automatic Defibrillator Implantation Trial (MADIT) II Patients. J Am Coll Cardiol, vol 44 (7), 2004, p. 1481-87.

[12] Ferreti Gf, R.E. L; Zayat, M; Mazzara, D; Rimatori, C; Pupita, G; Mannello, B; Russo, P. A New Method for the Simultaneous Measurement of the RR and QT Intervals in Ambulatory ECG 
으 Recordings. Computers in Cardiology, IEEE Computer Society, 1992, p.

으 171-174.

5

[13] Helfenbein, E. D; Zhou, S. H; Lindauer, J. M.; Field, D.Q.; Gregg, R.

○ E, Wang, J. J.; Kresge, S. S.; Michaud, F. P. An algorithm for continuous real-time QT interval monitoring. Journal of $\frac{1}{c}$ Electrocardiology, 39, 2006, p. 123-127.

[14] Daskalov, I.K.; Christov, I.I. Automatic detection of the N electrocardiogram T-wave end. Med. Biol. Eng. Comput. 37, 1999, p. \pm 348-353.

N [15] Zhang, Q.; Illanes Manriquez, A; Médigue, C.; Papelier, Y.; Sorine, $\widehat{o}$ M. An Algorithm for Robust and Efficient Location of T-Wave Ends in Electrocardiograms. IEEE Trans. Biomedical Engineering. 53, 2006, (D) p. 2544-2552.

[16] Last T, Nugent C.D, Owens F J. Multi-component based cross correlation beat detection in electrocardiogram analysis. Biomedical Engineering. Online, 26, 2004. Disponível em: http://www.biomedicalengineering-online.com/content/3/1/26.

[17] Vila, J.; Gang, Y.; Presedo, J.; Fernández-Delgado, M.; Barro, S.; Malik, M. A new approach for TU complex characterization. IEEE Trans. Biomed. Eng. 47, 2000, p. 764-772.

[18] Martinez, J.P.; Almeida, R.; Olmos, S. Rocha, A. P.; Laguna, P. A wavelet-Based ECG Delineator: Evaluation on Standard Databases. IEEE Trans. Biomedical Engineering. 51, 2004, p. 570-581.

[19] Vázquez Seisdedos, Carlos R. Análisis del ECG en monitorización ambulatoria para el diagnóstico cardíaco y la predicción de muerte cardíaca súbita. 2001, p. 1-327. Disponível em: http://www.tesisenxarxa.net/TDX-1105108-111710/. Acesso em: 11 out. 2011.

[20] Task Force of The European Society Of Cardiology And The North American Society Of Pacing And Electrophysiology. Heart rate variability. Standards of measurement, physiological interpretation, and clinical use. European Heart Journal, 17, 1996, p. 354-381.

[21] Vázquez-Seisdedos, CR, Suárez León AA, Evangelista Neto J. A Comparison of Different Classifiers Architectures for Electrocardiogram Artefacts Recognition, Lectures Notes on Computer Sciences 8259. p. 487 ff. J. Ruiz-Shulcloper and G. Sanniti di Baja (Eds.): CIARP 2013, Part II, LNCS 8259, pp. 254-261, 2013. () Springer-Verlag Berlin Heidelberg 2013.

[22] Vázquez-Seisdedos CR, Neto JE, Reyes E.J.M, Klautau A, Oliveira RCL. New approach for T-wave end detection on electrocardiogram: performance in noisy conditions. Biomedical Engineering. Online 2011; 10 (77): $1-11$. 